\title{
Fetal Exposure to Acetochlor Impaired Cognitive Function in Rats via Abnormal Gut Microbiome and Metabolic Disorders
}

\section{Wei Wei}

Harbin Medical University

Huanyu Wu

Harbin Medical University

Wenbo Gu

Harbin Medical University

Jiaxu Xu

Harbin Medical University

Cong $\mathrm{Hu}$

Harbin Medical University

Yifan Ma

Harbin Medical University

Guolian Qi

Harbin Medical University

Hongyan Jiang

Harbin Medical University

Yujia Long

Harbin Medical University

XiTao Jiang

Harbin Medical University

Tianshu Han

Harbin Medical University

Wenbo Jiang ( $\nabla 1615199541 @ q q . c o m)$

harbin medical university https://orcid.org/0000-0001-5885-5261

\section{Research}

Keywords: acetochlor, fetal exposure, gut microbiome, memory and cognition

Posted Date: November 22nd, 2021

DOI: https://doi.org/10.21203/rs.3.rs-1057583/v1 
License: (c) (i) This work is licensed under a Creative Commons Attribution 4.0 International License. Read Full License 


\section{Abstract}

Background: Current evidence has shown that acetochlor has adverse effects on health, which has been widely used in agricultural production, while the evidence on the effects of fetal acetochlor exposure on nervous system is quite limited. The fetus period is the most sensitive window period in the whole lifetime to external stimuli. Therefore, we established a rat model fetal exposed to acetochlor.

Results: Impaired cognitive function, abnormal gut microbiome and significant changes in the hippocampus and colon could be observed in the acetochlor-exposed group. We also observed remarkable impairment in the hippocampal cells in the histomorphology observation. Fecal microbiota transplantion(FMT) was performed to elucidate whether the gut microbiome mediated the acetochlorinduced abnormal behavior. Then the proteomics analysis and metabonomics analysis in the hippocampus indicated that differential expression proteins were mainly enriched in amino acid neurotransmitter-related pathways, long-term potentiation, and synaptic-related pathways and differential metabolite were mainly enriched in mutiple neurotransmitter-related pathways. Then we observed remarkable changes in the Camk2b/Erk1/2/NF-kB pathway and Prkaca/p-CREB1/BDNF pathway in the FMT group, which may cause abnormal cognitive behaviors.

Conclusions: Our results demonstrate that fetal exposure to acetochlor could induce abnormal behaviors in rats via disrupting the intestinal microbiome.

\section{Background}

Acetochlor, one of the amide selective preemergence herbicides, is widely used in agricultural production, which has the advantages of wide herbicidal spectrum, low price, convenient application, and outstanding effect[1-3]. Due to the wide use of acetochlor, surface water and soils has been contaminated which is a increasingly serious hazard to the public[4,5]. A previous research has showed that in the riparian soil of the Songhua River basin in China, the concerntration of acetochlamine in the sediment could be up to $11.76 \mathrm{mg} / \mathrm{kg}$, and the maximum could reach $709.37 \mathrm{mg} / \mathrm{kg}[6]$. Moreover, acetochlamine level in surface water in the midwest of the US has also reached $2.5 \mathrm{mg} / \mathrm{L}[7]$. These concentrations may be sufficient to have adverse effects on health who live in these environments.

Previous studies have demonstrated that residual acetochlor and its metabolites in the environment could be enriched in multiple organs through the food chain and drinking water contaminant, causing health problems[8-10]. As the fetal stage is the most sensitive window period to environmental disturbances, which could affect the development of intestinal tract. Numerous researches have documented that disturbed gut microbiota could further influence the structure and function of central nervous system, including the impairment of cognitive function[11,12]. Therefore, based on the evidence above, we proposed a hypothesis that fetal exposed to acetochlor could damage cognitive function via disturbing gut microbiota. This study, therefore, established a rat model of fetal acetochlor exposure to 
explore the potential effects of acetochlor on the hippocampus and elucidate the role of intestinal microbiota in the abnormal behaviors.

\section{Method}

The complete process of this study regarding 1)Rats and acetochlor treatment; 2)Behavioral experiment; 3)DNA extraction of fecal samples and amplicon sequencing; 4)Microbiome analysis; 5)Fecal microbiota transplant; 6)Proteomics analysis; 7)Metabonomics analysis; 8)Western blot analysis and real-time PCR assay; 9)Immunofluorescence; 10)Statistical analysis were provided as follows.

\section{Rats and acetochlor treatment}

Thirty eight-week-old rats were purchased from Vital River Lab Animal Technology Co., Ltd (Peking, China), and housed in a controlled environment room (12h:12h light/dark cycle, humidity (40\%-50\%) and constant temperature $\left(18-22^{\circ} \mathrm{C}\right)$ ) with food and water ad libitum. The study design was shown in Fig. $1 \mathrm{a}$. All the rats were randomly assigned to acclimate for one week and then mated. The female rats were examined for vaginal smears in the next day and housed individually once pregnant. The pregnant rats were then randomly divided into control group and two exposure groups $(5 \mathrm{mg} / \mathrm{kg}$ and $10 \mathrm{mg} / \mathrm{kg})$ with five rats in each group. Then the acetochlor exposure was orally conducted via oil before delivery. In our study, only male offspring were selected for further experiments. At 4-week age, the feces of offspring rats were collected for detection and fecal microbiota transplant experiments. At 8-week age, offspring rats were arranged for the behavioral tests. And then, they were anesthetized with phenobarbital sodium, and collected the blood, hippocampus and other tissues which were weighed and rapidly frozen in liquid nitrogen and stored at $-80{ }^{\circ} \mathrm{C}$ for further researches. All the animals experiments were approved by the Medical Ethics Committee of Harbin Medical University and were performed according to the Guide for Care experiment.

\section{Behavioral experiments}

\section{Morris water maze test}

The MWM test was performed based on the previous study[33]. In the training test, the rats were placed into the pool from four different quadrants and forced to swim four times each day for 5 days. The time allowed for the rats to reach the terrace was set as $1.5 \mathrm{~min}$. When the rats found the platform, allowed them to stay on the platform for 10 s and record the latency. The probe test was performed $24 \mathrm{~h}$ after the last acquisition trial. In the probe test, the platform was removed from the sink and performed a $90 \mathrm{~s}$ probe test. The number of each rats crossing the platform site in $90 \mathrm{~s}$ was recorded.

\section{Shuttle box test}

The shuttle box test was conducted based on the previous study[34]. Briefly, this test was performed on each rats for 5 consecutive days. Each test consists of a $10 \mathrm{~s}$ conditioned light stimulus followed by an unconditional stimulus of $10 \mathrm{~s}$ electric $\operatorname{shock}(0.5-1.0 \mathrm{~mA})$ at $20 \mathrm{~s}$ intervals. If the rats stepped into the 
other side of the shuttle box during the conditioned stimulus, the stimuli was turned off. And then recorded the latency of step-through.

\section{Step-down test}

The step-down test was performed based on the previous study[34]. Briefly, before the training session, the rats were placed on the platform. Once stepped down on the steel bars, the rats would received foot shocks and jumped back to the platform to avoid the shock. When they stepped down onto the bars,they may jump back immediately because of another shock. Retention test session was carried out $24 \mathrm{~h}$ after

the training, the rats were placed on the platform. No footshock was given and the step-down latency was recorded as an index of short-term memory retention.

\section{New object recognition test}

The new object recognition test was performed based on the previous study[35]. In the first phase, the rats were permitted to walk around without any objects for $10 \mathrm{~min}$. In the second phase, two identical objects(object A1 and A2) were placed in the active area of the rats for $5 \mathrm{~min}$. In the third phase, the cylindrical object(object $A$ ) was replaced by the square object(object $B$ ). The exploring duration of the field was recorded as EA1 and EA2, and the exploring duration of the new object was recorded as EA and EB. The discriminant index was calculated by the formula: EB-EA/(EA+EB). Once greater than 0.5 , the mice was considered to tend to explore new objects.

\section{DNA extraction of fecal samples and amplicon sequencing}

Feces of all offspring rats were collected under sterile conditions at the 4th week, and then were rapidly stored at $-80{ }^{\circ} \mathrm{C}$ for further study. Total DNA in feces was separated utilizing a QIAamp Fast DNA Stool Mini Kit(Qiagen, Germany). The PCR-amplified amplicon of the V3 and V4 hypervariable regions of the 16S rRNA gene was performed, and then were normalized by concentration and subjected to $300 \mathrm{bp}$ paired-end sequencing on an Illumina MiSeq platform(Illumina, San Diego, USA). Raw fastq files were used for demultiplex and quality filtering with trimmomatic, and then merged with FLASH software.

\section{Microbiome analysis}

The calculation of richness estimations of the OTUs were performed by QIIME 1.9.1 64-bit. Ace, Shannon, Chao1, and Simpson indices were calculated to identify the alpha diversity of OTU. PCoA was performed to demonstrate the clustering of different samples. The weighted UniFrac distance was utilized to quantify differences in community composition. Linear discriminant analysis(LDA>2) was identified the characteristics that caused the greatest difference between the control and acetochlor groups. Biological function categorization was predicted and annotated with KEGG pathways based on the PICRUSt analysis. All the procedure of sequencing was conducted by the majorbio company, and the analysis was performed on an online website https://cloud.majorbio.com. 


\section{Fecal microbiota transplant}

The transplant of fecal was modified based on an established protocol. Briefly, for antibiotic treatment, the rats were given the gavage of $100 \mathrm{mg} / \mathrm{kg}$ metronidazole, and combination of $1 \mathrm{~g} / \mathrm{L}$ ampicillin and neomicin, $0.5 \mathrm{~g} / \mathrm{L}$ vancomycin in drinking water for one week[36]. Then the $5 \mathrm{~g}$ feces collected from 4week rats were transferred to antibiotic-treated recipient rats. The feces from donor rats were resuspended in $50 \mathrm{ml}$ sterile PBS. And the suspension was then vigorously mixed for $60 \mathrm{~s}$ and centrifuged(500 g, $3 \mathrm{~min})$. After filtration, the supernatant was collected and prepared on the same day of transplantation within 10 min before oral gavage. The recipient rats was achieved by gavage with $1 \mathrm{~mL}$ of the supernatant once a a day until 8-week old.

\section{Proteomics analysis}

The hippocampus of recipient-rat was collected and prepared for proteomic analysis to identify the differential protein expressions using iTRAQ-LC-MS/MS. $100 \mathrm{mg}$ hippocampus sample was suspended in $100 \mu$ tetraethyl-ammonium bromide and tryptic digested with 1:50 Trypsin Gold(Promega, Madison, WI, USA). The suspension was homogenized by vortexing for $3 \mathrm{~min}$ and centrifugated at 13,000 rpm for 10 min. The supernatant was then collected and measured by the BCA protein assay(Thermo Scientific, Waltham, MA). Peptide labeling was conducted with an iTRAQ Reagent 8-plex Kit following the manufacturers' protocol. The peptide mixture was separated at high $\mathrm{pH}$ and each fraction dried in a vacuum concentrator for the next step. The separation was conducted using a nano-HPLC instrument and subjected to tandem mass spectrometry for data-dependent acquisition detection by nanoelectrospray ionization. All differential expression proteins were identified with a $1 \%$ false discovery rate (FDR) and further enriched for GO and KEGG analysis by using the DAVID bioinformatics database (https://david.ncifcrf.gov/).

\section{Metabonomics analysis}

Hippocampus samples(50 mg) were homogenized in dichloromethane/ methanolwater(3:1). After centrifugation, the supernatants were collected in Eppendorf tubes. The remaining pellets were homogenized in methanol/water(1:1). Then the homogenates were centrifuged and Supernatants were collected and dried in a fume cupboard. The extracts were resuspended in $120 \mathrm{~mL}$ of acetonitrile/water(3:1) for further UPLCeQTOF/MS analysis. Use Waters ACQUITY UPLC system and Waters Micromass Q-TOF micro ${ }^{\text {TM }}$ mass spectrometer(ESI) to perform UPLCeQ-TOF/MS analysis in both positive and negative modes(Waters Corporation, USA). Inject $2 \mathrm{ml}$ of the hippocampus samples into the ACQUITY UPLC ${ }^{\text {TM }}$ BEH C18 column. Use marklynx Application Manager 4.1 to process the raw data files generated by UPLCeQ-TOF/MS, and calibrate them according to our previously published agreement.

\section{Western blot analysis and real-time PCR assay}


For western blot analysis, the total protein was extracted by RIPA lysis buffer(Beyotime, Shanghai) plus phenylmethanesulfonyl fluoride(Beyotime, Shanghai) and denatured by heating. Then the protein was separated by SDS-PAGE gel electrophoresis on $10 \%$ gels and transferred to PVDF membranes using a tank transfer system at $200 \mathrm{~mA}$ in Tris-glycine buffer at $4{ }^{\circ} \mathrm{C}$. The membrane was blocked with a blocking buffer(containing $5 \%$ milk and $0.1 \%$ Tween 20 TBS). Next, the membranes were incubated with diluted primary antibodies overnight. The following antibodies were used for western blotting:Prkaca(1:2000,abcam), Camk2b(1:1500,abcam), p-Erk1/2(1:1200,abcam), NFKB(1:1000,abcam), p-CREB (1:1000,abcam), BDNF(1:1200,abcam). The membranes were washed three times with TBST(containing $0.1 \%$ Tween 20 TBS) for 10 min each. We incubated secondary antibodies against rabbit $\operatorname{lgG}(1: 7500)$ for $60 \mathrm{~min}$. Then, the membranes were washed thrice with TBST and exposed in a dark room. The signal was detected by using an ECL kit in the ratio of $A: B=1: 1$.

For the real-time PCR, the total RNA of hippocampus was isolated by TRIZOL reagent(Invitrogen, Carlsbad, CA). RNA was reverse transcribed to CDNA with a high-capacity CDNA reverse transcription kit(Applied Biosystems, USA). The expressions of mRNA were determined by the SYBR Green PCR Master Mix and the 7500 FAST Real-time PCR System(Applied Biosystems, USA). All primers of each targeted gene were synthesized at Invitrogen, and the sequences are listed in Supplementary table1. And then using $2^{-\Delta \Delta C t}$ method to analyze the relative levels of mRNAs.

\section{Immunofluorescence}

The sections were blocked using $10 \%$ goat serum for 40 min and then incubated with anti-p-CREB(diluted 1:150; abcam, USA) and anti-p-Erk1/2 antibody(1:150; Invitrogen, USA) for $2 \mathrm{~h}$ at room temperature. And then, the sections were incubated with Alexa Fluor 488-conjugated anti-rabbit IgG secondary antibody (1:1000, Waltham, USA.) mixing with antibody dilution fluid for $45 \mathrm{~min}$ after washed three times. Counterstaining was performed with the fluorescent DNA stain 4'6-diamidino-2-phenylindole(DAPI) for 15 min. The sections were observed using a laser scanning confocal microscope(A1R/A1; Nikon, Japan).

\section{Statistical analysis}

Statistics on alpha diversity and organism level microbiome phenotypes were performed based on MannWhitney test. The differences between all raw obtained values(mean $\pm S E$ ) were analyzed by a one-way ANOVA analysis or unpaired Student's t test using SPSS v21.0 software(IBM, USA). A two-sided pvalue $<0.05$ was considered to be statistically significant.

\section{Results}

\section{Effect of acetochlor exposure on maternal and offspring rats}


The number of live pups in the acetochlor groups was significantly reduced compared with the control( $P<0.01$, Fig. $1 \mathrm{~b})$. The organ coefficients of liver and brain were significantly decreased in the acetochlor group $(P<0.01$, Fig.1c), whereas the others did not differ in offspring rats(all the $P>0.05$, Fig.1c). The length of colon was remarkably decreased in the acetochlor group $(P<0.01$, Fig. $1 \mathrm{~d})$, while the length of the small intestine, cecum and rectum were not differ(all the $P>0.05$, Fig. $1 \mathrm{~d}$ ). There were no differences in body weight between the control pups and the acetochlor pups as well as the food intake $(P=0.25$, Fig.1e; $P=0.11$, Fig.1f; $P=0.23$, Fig.1g).

\section{Abnormal neurobehavior and hippocampal histomorphology induced by acetochlor exposure}

For morris water maze(MWM) test, the escape latency and the mean distance to the platform were remarkably increased $(P<0.01$, Fig.2a; $P<0.01$, Fig.2b), while the total number of platform area crossings was significantly decreased in the acetochlor-exposed group compared to the control( $P<0.01$, Fig. $2 \mathrm{c})$, which indicated the impaired long-term spatial memory. For shuttle box test, the acetochlor groups showed a significant decrease in the latency of step-through compared to the control $(P<0.01$, Fig. $2 \mathrm{~d})$. For step-down test, the latency of step-down and the error frequencies in the acetochlor groups were significantly decreased compared with the control( $P<0.05, P<0.01$, Fig.2e; $P<0.05, P<0.01$, Fig.2f). For new object recognition test, in the first phase, there was no difference in the exploration time in each group $(P>0.05$, Fig.2g). In the second phase, acetochlor groups rat presented significantly lower preference ratio compared to the control( $P<0.05$, Fig. $2 \mathrm{~h})$. Besides, the results of $\mathrm{HE}$ staining showed the acetochlor group rat had an irregular, sparse arrangement of hippocampal neuron cells and shrunken, darkened nuclei(Fig.2i).

\section{Abnormal gut microbiome composition and colonic histomorphology in offspring rats}

Principal coordinates analysis(PCoA) plots on the operational taxonomic units(OTU) level revealed a distinct separation of fecal microbiome between the control and $10 \mathrm{mg} / \mathrm{kg}$ group, which passed the Adonis analysis ( $\mathrm{R}=0.2698, P=0.003$, Fig.3a). Detailed microbial composition on the phylum level and genus level were presented in Fig.3b,c. For the alpha diversity, the ace indices, chao1 indices, and shannon indices were dramatically decreased in the $10 \mathrm{mg} / \mathrm{kg}$ group $(P<0.001, P<0.001, P<0.05$, Fig.3e$3 \mathrm{~g}$ ), whereas simpson index was not significantly changed( $P>0.05$, Fig.3h). And the LEfSe analysis indicated that all the altered genera could be identified as the biological markers to distinguish the control and $10 \mathrm{mg} / \mathrm{kg}$ group, including firmicutes and acinetobacter which have been reported to be associated with mental disorders(Fig.3d,i). Besides, the results of HE staining showed that the colon tissues structure of the acetochlor groups were disordered, and there was serious infiltration of inflammatory cell in the intestinal mucosa and submucosa. And intestinal villi were reduced in height and number, and the tight connections between cells was obviously damaged(Fig.3j).

\section{Abnormal neurobehavior and hippocampal histomorphology in FMT group}


After feces suspension of the $10 \mathrm{mg} / \mathrm{kg}$ group was transplanted into the control of offspring rats, behavioral experiments and morphological observation were performed again. For MWM test, compared to the control group, the escape latency and the mean distance to the platform were significantly increased $(P<0.05$, Fig.4a; $P<0.01$, Fig.4b), while platform area crossing times were decreased in the FMT group ( $P<0.01$, Fig.4c). For shuttle box test, compared to the control group, the FMT group rat presented a remarkable decrease in the latency of step-through $(P<0.01$, Fig.4d). For step-down test, the latency of step-down and the error frequencies in the FMT group were significantly decreased compared with the control ( $P<0.01$, Fig.4e; $P<0.01$, Fig.4f). For new object recognition test, in the first phase, there were no differences in the exploration time in both groups ( $P>0.05$, Fig.4g). In the second phase, FMT group rat showed prominently lower discrimination ratio compared with the control $(P<0.01$, Fig.4h). Besides, the results of HE staining showed hippocampus neuron cell bodies were arranged in a compact cell layer and the cell nucleoli and cytoplasm were distinctly and clearly in the control group. However, The neuron cells were irregular in shape and contained less cytoplasm in the FMT group(Fig.4i).

\section{Abnormal gut microbiome composition and colonic histomorphology in FMT group}

PcoA plots on the OTU level showed a distinct separation of fecal microbiome between the control and FMT group, and also among the four groups (control group1, acetochlor group $(10 \mathrm{mg} / \mathrm{kg}$ ), control group2, FMT group), which had passed the Adonis analysis ( $\mathrm{R}=0.2698, P=0.003$, Fig.5a; $\mathrm{R}=0.3334$, $P=0.001$, Fig. $5 b)$. The microbial composition on the phylum level and genus level were presented in Fig.5c-5d. Whereas the ace indices, chao 1 indices, shannon indices, and simpson index were not differential in the FMT group(All the $P>0.05$, Fig.5d-g). And the LEfSe analysis showed that differential genera could be regarded as the biological markers to distinguish the control and FMT group(Fig.5h,i). Moreover, the results of HE staining indicated that the colon tissues structure of the FMT group were severely damaged with inflammatory cell infiltrating in the intestinal mucosa and submucosa(Fig.5j).

Besides, we found that there are 29 mutual differential signaling pathway in both gut bacteria analysis before and after transplantation, including neurotransmitter-related pathways, such as phenylalanine, tyrosine and tryptophan biosynthesis, alanine, aspartate and glutamate metabolism(Fig.6a). And figure 6 showed the relationships of common differential pathways with the top 50 differential flora in abundance before and after transplantation(Fig.6b,c).

\section{Hippocampus metabolomics profiles in FMT group}

To explore the differences in the metabolic profiles of the control and FMT groups, the PLS-DA models were performed. PLS-DA scores plots showed a significant discrepancy in the hippocampus metabolic profiles between control and FMT groups(Fig.7a,b). The volcano map shows the overall perspective of the different metabolites in the positive and negative ion mode(Fig.7c,d, Supplementary table2). And the differential metabolites were enriched in the mutiple neurotransmitter-related pathways, including glycine, serine and threonine metabolism, sphingolipid metabolism, glutamate metabolism, steroid hormone biosynthesis(Fig.7e, Supplementary table3). 


\section{Differential protein expressions in rats hippocampus induced by FMT}

To elucidate how the acetochlor damaged cognitive and memory function via disturbing gut microbiome in rats, a proteomic analysis was performed in the rats hippocampus. In the FMT group, we found 531 differential protein expressions, including 231 down-regulated and 300 up-regulated proteins as compared to the control(Fig.8a). And Gene Ontology(GO) analysis results indicated that differentially expressed proteins were enriched in synaptic vesicle endocytosis, cellular response to nerve growth factor, neuron development, regulation of short-term neuronal synapse(Fig.8b). Enrichment plots obtained by the Gene Set Enrichment Analysis(GSEA) showed that the majority of the neuron differentiation, neuron development, regulation of axonogenesis and synapic vesicle priming-associated genes were downregulated in FMT group when comparing with the control group(Fig.8c-f). Kyoto Encyclopedia of Genes and Genomes(KEGG) pathway analysis revealed that differentially expressed proteins were mainly enriched in metabolic pathways(tryptophan metabolism; alanine, aspartate and glutamate metabolism), signaling pathways(long-term potentiation; MAPK signaling pathway), synaptic-related pathways(Fig.8g). S-shape figure showed the proteins(Prkaca, Camk2B) with greater fold-change.

Consistently, compared with the control group, staining for the p-Erk1/2(Fig.9a-9d) and p-CREB1(Fig.9e9h) was significantly changed in the FMT group. Besides, compared with the control, the mRNA levels of Prkaca, BDNF in the FMT group were significantly down-regulated $52.67 \%$ and $50.12 \%$, whereas Camk2b and NF-KB mRNA levels were significantly up-regulated $75.43 \%$ and $95.25 \%$ (Fig.9j-9m). And the protein expression levels of Prkaca, p-CREB1 and BDNF were also remarkably reduced $51.27 \%, 63.29 \%, 50.39 \%$ in the FMT group, whereas Camk2b, p-Erk1/2 and NF-kB protein levels were significantly increased $113.22 \%$, $70.87 \%$ and $82.33 \%$ (Fig.9n-9s). All the representative bands were presented in Fig.9i.

\section{Discussion}

This study firstly demonstrated that fetal acetochlor exposure was associated with impaired cognitive function which may induce by the aberrant gut microbiome composition and function. Moreover, the obviously abnormal metabolic profile and protein expression profile in hippocampus were also identified after fecal microbiota transplantation, in which Camk2b/Erk1/2/NF-kB pathway and Prkaca/p-CREB1/ BDNF pathway may make a great contribution. To the best of our knowledge, our study is the first one to establish the association between fetal acetochlor exposure and abnormal neurobehavioral alteration, and to elucidate whether and how the gut microbiome mediated the association.

The most important discovery in our present study is that fetal exposure to acetochlor could induce abnormal behavior in adulthood. Our behavioral experiments demonstrated that acetochlor-exposed rats displayed impaired, cognitive function, dimensional perception, conditioned avoidance learning and short-term and long-term memory. The previous studies have shown that acetochlor exposure could affect T3-mediated behavioral changes in Rana catesbeiana tadpoles during the development period[1315]. This is due to the time of first stop and total time spent immobile becoming more and more larval- 
like. Our observations in neurological behavior and hippocampal histomorphology could be partially demonstrated by these findings.

Meanwhile, firmicutes, tenericutes, deinococcus-thermus and bacteroidetes were the dominant bacteria at the phylum level, and significant differences in the constitution and function of these gut microbes could be found in acetochlor-exposed group. As we all known, in the fetal period, the structure of the fetus's intestinal bacteria is largely determined by the mother's[16, 17]. The changes in the structure and function of the intestinal microflora of the offspring rats were probably due to the effects of acetochlor on the maternal intestinal microflora and vaginal microflora[18, 19]. Exposure to acetochlor during the critical period of colonization and stabilization of intestinal flora undoubtedly affected the ratio of beneficial and harmful bacteria in offspring rats, even resulting in the production of a series of harmful co-metabolites. The previous study demonstrated that residual acetochlor in soil conferred a long-term impairment on fungal community which could partially support our findings[20]. Further, it is well documented that the complex interplay between the gut microbiome and brain function has been identified as the gut-brain axis $[21,22]$. The gut microbiome could bi-directionally communicate with the central nervous system and affect anxiety, pain, cognition, and mood[23-26], which may partially support the impact of acetochlor exposure on the impairment of brain tissue.

To elucidate whether the gut microbiome mediated the acetochlor-induced abnormal behavior, we performed fecal microbiota transplant experiments at 4th week. This time point is a good reflection of the early intestinal microbiota because of the necessary adaptation after weaning at 3 weeks of age[27, 28]. Meanwhile, at this time point, the hippocampus is considerably sensitive and vulnerable to environmental stimuli[29]. Our results have shown that transplanting feces from acetochlor-exposed rats into recipient rats could mostly reproduce the phenotypes observed in the acetochlor-exposed rats. The poor performance of FMT rats in the behavioral experiments and may suggest that disturbed gut microbiome could directly induce abnormal behavior. Meanwhile, noticeable alterations of histomorphology in the hippocampus could be readily detected in the FMT rats, which is consistent with the result of behavioral tests. Combined these results above, we could reach a reliable conclusion that early life acetochlor exposure could impair memory and cognitive function through the disturbed gut microbiome.

Furthermore, we focused on the differential metabolites and differential expressed proteins in the hippocampus to disclose how the disturbed gut microbiome affected the function of the hippocampus. The metabolic profile showed that aromatic amino acid biosynthesis and alanine, aspartate, glutamate metabolism, and sphingolipid metabolism were significantly changed which could regulate local and systemic immune metabolism, neuronal responses, excitatory/inhibitory neurotransmitter biosynthesis in the host. These could also be found in the mutual differential pathways in both donor and recipient rats. These consistent results indicated that acetochlor exposure induced the disturbed metabolites profile in the hippocampus via damaging the gut microbiota. Further, the proteomics and related validation experiments have showed that the Camk2b/p-Erk1/2/NF-kB pathway and Prkaca/p-CREB1/BDNF pathway were identified due to the significant alteration. It is well conducted that Camk2b is a member of Calmodulin-dependent protein kinase (CaMK) family, and its activation could further promote Erk1/2 
phosphorylation, resulting in activation of inflammatory pathways. The neuroinflammatory molecules could lead to indirect/direct neuronal toxicity, which may contribute to neurodegenerative disorders[30]. Besides, our results also showed that acetochlor exposure could elevate prkaca expression and active CREB1 phosphorylation, and thus regulate BDNF level related to neuronal plasticity[31]. In a recent research, BDNF, together with its upstream regulator CREB1, were down-regulated by paraquat exposure in rats which is another kind of common herbicide extensively used in rice or cereal fields[32], which could partially support our findings.

Our study should be considered alongside a limitation. We have confirmed these changes in the microbial function and construction, but not enough to conclude mechanism of virulence is due to the interaction between specific gut microbiota or its metabolites with the development of the hippocampus. Therefore, future studies should be further performed to elucidate the role of specific bacteria-generated metabolites on the hippocampus and to perfect the risk evaluation of acetochlor exposure in human beings. Meanwhile, the observations in our study had several important implications. Some pregnant women would like to stick to engage in farm work in the early stages of pregnancy, and long-term unconscious herbicide exposure has become a major threat to the health of their offspring. Our research may raise awareness of the protection of pregnant women in families using herbicides. Besides, our results suggested that the gut-brain axis may play a crucial role in the neurotoxicity of acetochlor. Therefore, intake more foods that could help keep the gut bacteria healthy may be beneficial to reverse the adverse effect of gestational exposure to acetochlor.

\section{Conclusion}

The present study added evidence regarding the effects of fetal acetochlor exposure on gut microbiota and neurological behavior in rats. We found that fetal exposure to acetochlor could disturb the gut microbiome and hippocampal metabolites, and thereby induce abnormal behavior in rats via activating the Camk2b/p-Erk1/2/NF-kB pathway and Prkaca/p-CREB1/BDNF pathway.

\section{Abbreviations}

FMT: Fecal microbiota transplantion; MWM: morris water maze; OTU: operational taxonomic units; GO: Gene Ontology; GSEA: Gene Set Enrichment Analysis; KEGG: Kyoto Encyclopedia of Genes and Genomes; CaMK: Calmodulin-dependent protein kinase; PCoA: Principal coordinates analysis; DAPI: 4'6-diamidino-2phenylindole.

\section{Declarations}

\section{Acknowledgements}

We thank Xiaotian Chen (Fudan university) for technical assistance and critical reading of this manuscript 


\section{Author contributions}

WB Jiang, WW, and TS Han conceived the idea for the study, HY Wu and WB Gu were involved in the behavioral experiment. WB Jiang, XT Jiang, JX Xu, and C Hu were involved in the analysis of data. WB Jiang wrote the manuscript. YJ Long and YF Ma were responsible for analyzing the chip data. All the authors were responsible for revising the manuscript and approved the final version.

\section{Funding}

This work was supported by HMU Marshal Initiative Funding (HMUMIF-21011 to Wenbo Jiang), the National Natural Science Foundation of China (81803227 to Tianshu Han), the Young Elite Scientists Sponsorship Program by CAST (2019QNRC001 to Tianshu Han).

\section{Availability of data and materials}

The data supporting the findings of our study are available from the corresponding author upon request.

\section{Ethics approval and consent to participate}

All the animals experiments were approved by the Medical Ethics Committee of Harbin Medical University and were performed according to the Guide for Care experiment.

\section{Consent for publication}

Not applicable.

\section{Competing interests}

The authors declare that they have no competing interests.

\section{Author details}

${ }^{1}$ National Key Discipline, Department of Nutrition and Food Hygiene, School of Public Health, Harbin Medical University, Harbin, Heilongjiang Province 150081, P. R. China.

${ }^{2}$ Department of Pharmacology, College of Pharmacy Key Laboratory of Cardiovascular Research, Ministry of Education, Harbin Medical University, Heilongjiang, China.

${ }^{3}$ IT and Environment, College of Engineering, Charles Darwin University, Darwin, Northern Territory 0810, Australia. 
${ }^{4}$ Department of Cardiology, the First Affiliated Hospital of Harbin Medical University, Harbin, China.

\section{References}

1. Liu J, Zhang $X, X u$ J, et al. Anaerobic biodegradation of acetochlor by acclimated sludge and its anaerobic catabolic pathway. Sci Total Environ. 2020;748:141122.

2. Huang $T$, Huang $Y$, Huang $Y$, Yang $Y$, Zhao $Y$, Martyniuk CJ. Toxicity assessment of the herbicide acetochlor in the human liver carcinoma (HepG2) cell line. Chemosphere. 2020;243:125345.

3. Zhang Y, Xue W, Long R, Yang H, Wei W. Acetochlor affects zebrafish ovarian development by producing estrogen effects and inducing oxidative stress. Environ Sci Pollut Res Int. 2020;27(22):27688-27696.

4. Lerro CC, Koutros S, Andreotti G, et al. Use of acetochlor and cancer incidence in the Agricultural Health Study. Int J Cancer. 2015;137(5):1167-1175.

5. Wang H, Meng Z, Zhou L, et al. Effects of acetochlor on neurogenesis and behaviour in zebrafish at early developmental stages. Chemosphere. 2019;220:954-964.

6. Sun, X., Zhou, Q., Ren, W., Li, X., Ren, L., 2011. Spatial and temporal distribution of acetochlor in sediments and riparian soils of the Songhua River Basin in northeastern China. J. Environ. Sci. 23, $1684 \mathrm{e} 1690$.

7. Hladik, M.L., Bouwer, E.J., Roberts, A.L., 2008. Neutral chloroacetamide herbicide degradates and related compounds in Midwestern United States drinking water sources. Sci. Total Environ. 390, $155 \mathrm{e} 165$.

8. Taylor JP, Mills MS, Burns RG. Dissipation of acetochlor and its distribution in surface and subsurface soil fractions during laboratory incubations. Pest Manag Sci. 2005;61(6):539-548.

9. Barr DB, Hines CJ, Olsson AO, et al. Identification of human urinary metabolites of acetochlor in exposed herbicide applicators by high-performance liquid chromatography-tandem mass spectrometry. J Expo Sci Environ Epidemiol. 2007;17(6):559-566.

10. Chu C, Liu B, Liu J, et al. Phytoremediation of acetochlor residue by transgenic Arabidopsis expressing the acetochlor N-dealkylase from Sphingomonas wittichii DC-6. Sci Total Environ. 2020;728:138687.

11. Liu, Zhigang et al. "Gut microbiota mediates intermittent-fasting alleviation of diabetes-induced cognitive impairment." Nature communications vol. 11,1 855. 18 Feb. 2020.

12. Jiang, Chunmei et al. "The Gut Microbiota and Alzheimer's Disease." Journal of Alzheimer's disease : JAD vol. 58,1 (2017): 1-15.

13. eldhoen N, Helbing CC. Detection of environmental endocrine-disruptor effects on gene expression in live Rana catesbeiana tadpoles using a tail fin biopsy technique. Environ Toxicol Chem. 2001;20(12):2704-2708.

14. Hinther A, Domanski D, Vawda S, Helbing CC. C-fin: a cultured frog tadpole tail fin biopsy approach for detection of thyroid hormone-disrupting chemicals. Environ Toxicol Chem. 2010;29(2):380-388. 
15. Helbing CC, Ovaska K, Ji L. Evaluation of the effect of acetochlor on thyroid hormone receptor gene expression in the brain and behavior of Rana catesbeiana tadpoles. Aquat Toxicol. 2006;80(1):42-51.

16. Mei C, Yang W, Wei X, Wu K, Huang D. The Unique Microbiome and Innate Immunity During Pregnancy. Front Immunol. 2019 Dec 17;10:2886.

17. Al Nabhani Z, Eberl G. Imprinting of the immune system by the microbiota early in life. Mucosal Immunol. 2020 Mar;13(2):183-189.

18. Younge N, McCann JR, Ballard J, Plunkett C, Akhtar S, Araújo-Pérez F, Murtha A, Brandon D, Seed PC. Fetal exposure to the maternal microbiota in humans and mice. JCl Insight. 2019 Oct 3;4(19):e127806.

19. Gohir W, Kennedy KM, Wallace JG, Saoi M, Bellissimo CJ, Britz-McKibbin P, Petrik JJ, Surette MG, Sloboda DM. High-fat diet intake modulates maternal intestinal adaptations to pregnancy and results in placental hypoxia, as well as altered fetal gut barrier proteins and immune markers. $J$ Physiol. 2019 Jun;597(12):3029-3051.

20. Bai Z, Xu HJ, He HB, Zheng LC, Zhang XD. Alterations of microbial populations and composition in the rhizosphere and bulk soil as affected by residual acetochlor. Environ Sci Pollut Res Int. 2013 Jan;20(1):369-79.

21. Quigley EMM. Microbiota-Brain-Gut Axis and Neurodegenerative Diseases. Curr Neurol Neurosci Rep. 2017;17(12):94. Published 2017 Oct 17.

22. Dinan TG, Cryan JF. The Microbiome-Gut-Brain Axis in Health and Disease. Gastroenterol Clin North Am. 2017;46(1):77-89.

23. Mohajeri MH, La Fata G, Steinert RE, Weber P. Relationship between the gut microbiome and brain function. Nutr Rev. 2018;76(7):481-496.

24. Peirce JM, Alviña K. The role of inflammation and the gut microbiome in depression and anxiety. J Neurosci Res. 2019;97(10):1223-1241.

25. Lach G, Schellekens H, Dinan TG, Cryan JF. Anxiety, Depression, and the Microbiome: A Role for Gut Peptides. Neurotherapeutics. 2018;15(1):36-59.

26. Foster JA, McVey Neufeld KA. Gut-brain axis: how the microbiome influences anxiety and depression. Trends Neurosci. 2013;36(5):305-312.

27. Dong T, Guan Q, Hu W, et al. Prenatal exposure to glufosinate ammonium disturbs gut microbiome and induces behavioral abnormalities in rats. J Hazard Mater. 2020;389:122152.

28. Halpern R, Coelho R. Excessive crying in infants. J Pediatr (Rio J). 2016 May-Jun;92(3 Suppl 1):S405.

29. Chaudhury S, Nag TC, Jain S, Wadhwa S. Role of sound stimulation in reprogramming brain connectivity. J Biosci. 2013 Sep;38(3):605-14.

30. Herring BE, Nicoll RA. Long-Term Potentiation: From CaMKII to AMPA Receptor Trafficking. Annu Rev Physiol. 2016;78:351-65. 
31. Cammarota M, Bevilaqua LR, Medina JH, Izquierdo I. ERK1/2 and CaMKII-mediated events in memory formation: is 5HT regulation involved? Behav Brain Res. 2008 Dec 16;195(1):120-8.

32. Li B, He X, Sun Y, Li B. Developmental exposure to paraquat and maneb can impair cognition, learning and memory in Sprague-Dawley rats. Mol Biosyst. 2016 Oct 20;12(10):3088-97.

33. Jiang W, Li B, Chen Y, Gao S. The toxic influence of dibromoacetic acid on the hippocampus and prefrontal cortex of rat: involvement of neuroinflammation response and oxidative stress. Metab Brain Dis. 2017 Dec;32(6):2009-2019.

34. Jiang W, Chen Y, Li B, Gao S. DBA-induced caspase-3-dependent apoptosis occurs through mitochondrial translocation of cyt-c in the rat hippocampus. Mol Biosyst. 2017 Aug 22;13(9):18631873.

35. Cole E, Simundic A, Mossa FP, Mumby DG. Assessing object-recognition memory in rats: Pitfalls of the existent tasks and the advantages of a new test. Learn Behav. 2019 Jun;47(2):141-155.

36. Zhang L, Zhou W, Zhan L, Hou S, Zhao C, Bi T, Lu X. Fecal microbiota transplantation alters the susceptibility of obese rats to type 2 diabetes mellitus. Aging (Albany NY). 2020 Sep 12;12(17):17480-17502.

\section{Figures}


a
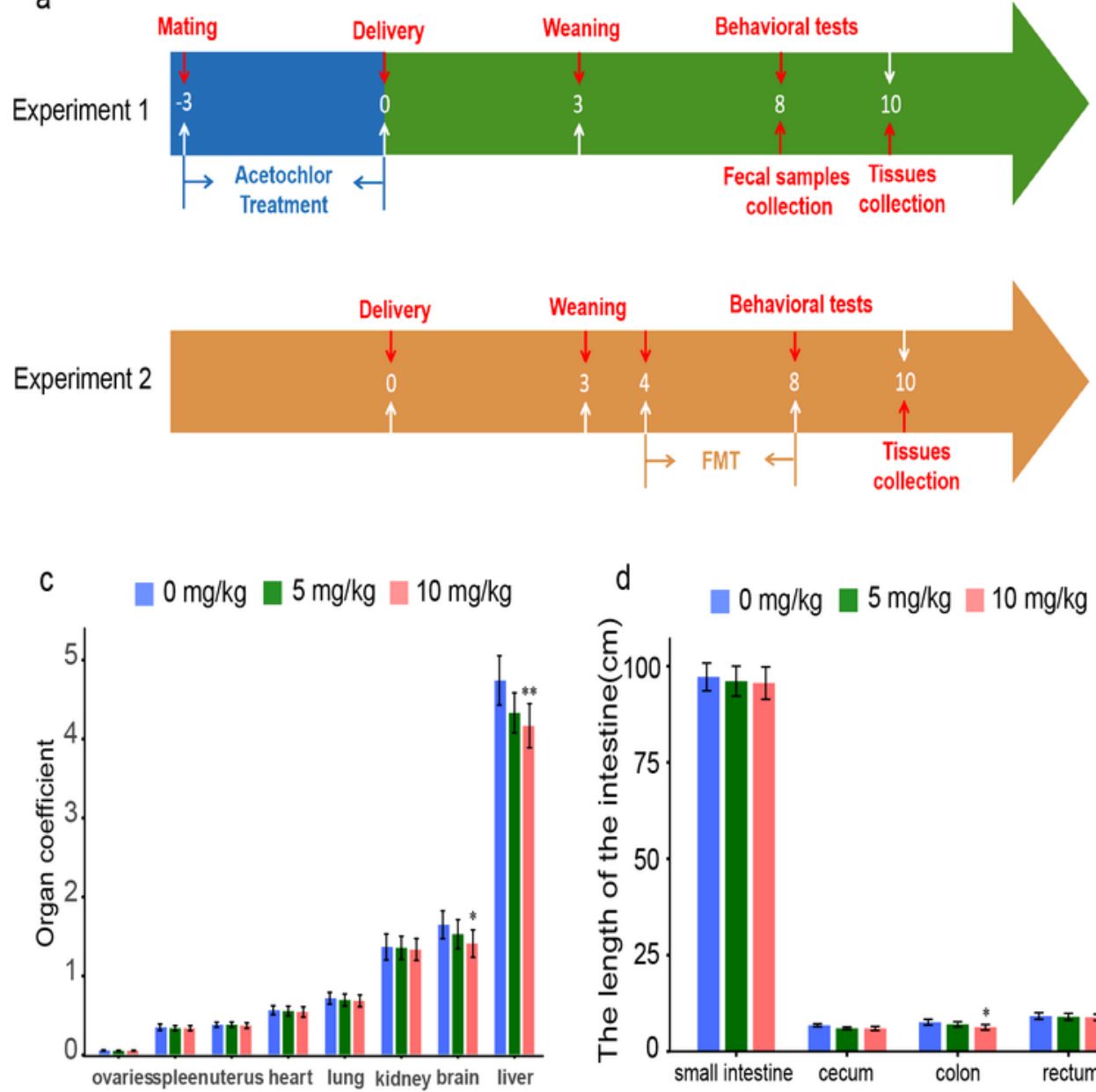

b 官 $0 \mathrm{mg} / \mathrm{kg}$ 安 $5 \mathrm{mg} / \mathrm{kg}$ 市 $10 \mathrm{mg} / \mathrm{kg}$

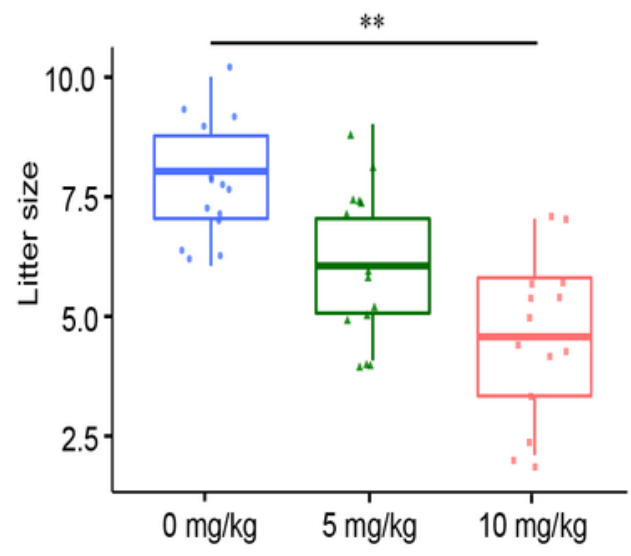

e

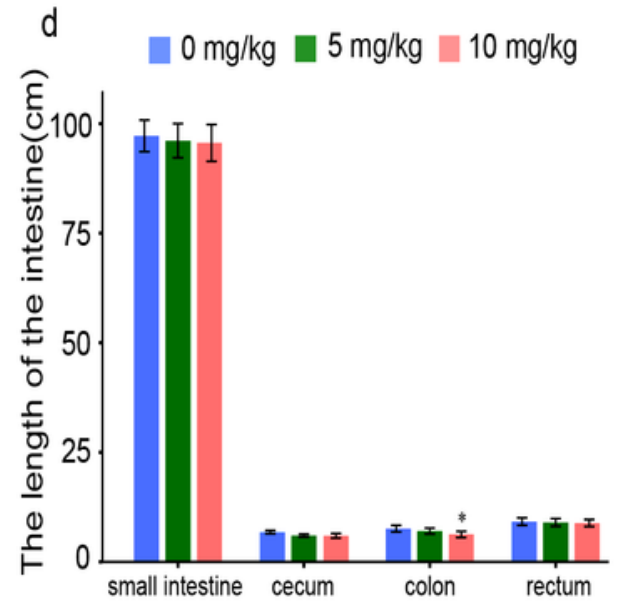

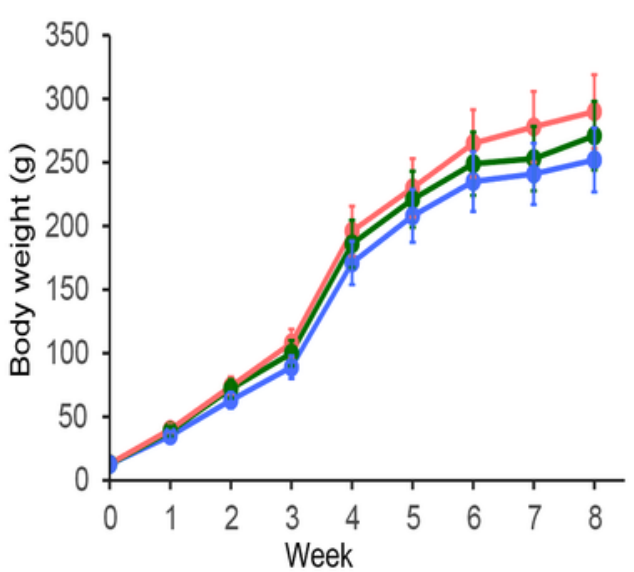

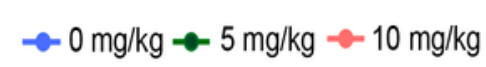

$\mathrm{f} \quad \rightarrow 0 \mathrm{mg} / \mathrm{kg} \rightarrow 5 \mathrm{mg} / \mathrm{kg} \leftrightarrow 10 \mathrm{mg} / \mathrm{kg}$ $\mathrm{g} \quad \rightarrow 0 \mathrm{mg} / \mathrm{kg} \rightarrow 5 \mathrm{mg} / \mathrm{kg} \rightarrow 10 \mathrm{mg} / \mathrm{kg}$
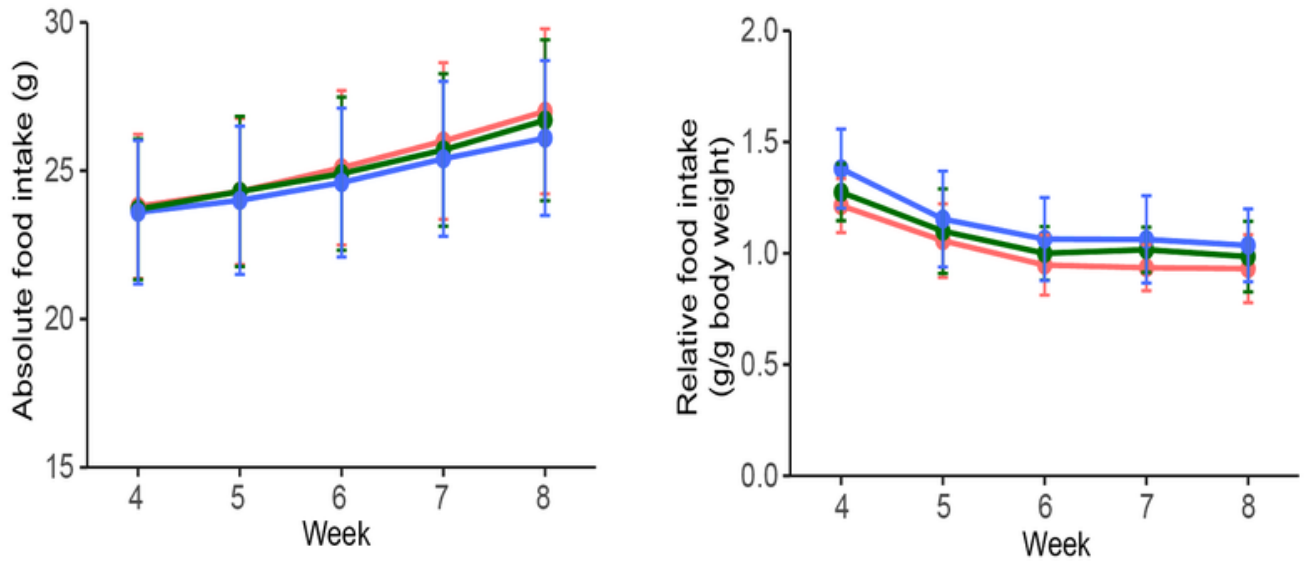

\section{Figure 1}

Effects of acetochlor exposure on offspring rats. (a)Overall experiment flow chart. (b)Effect of acetochlor exposure on the litter size. (c)Organ coefficient of offspring rats. (d)Effect of acetochlor exposure on the length of small intestine, cecum, colon and rectum. (e)The bodyweight of the offspring rats was not influenced by acetochlor. (f)Changes in absolute food intake during acetochlor exposure. (g)Changes in relative food intake during acetochlor exposure. Data were presented as mean $\pm S D\left({ }^{*}<<0.05\right)$ 

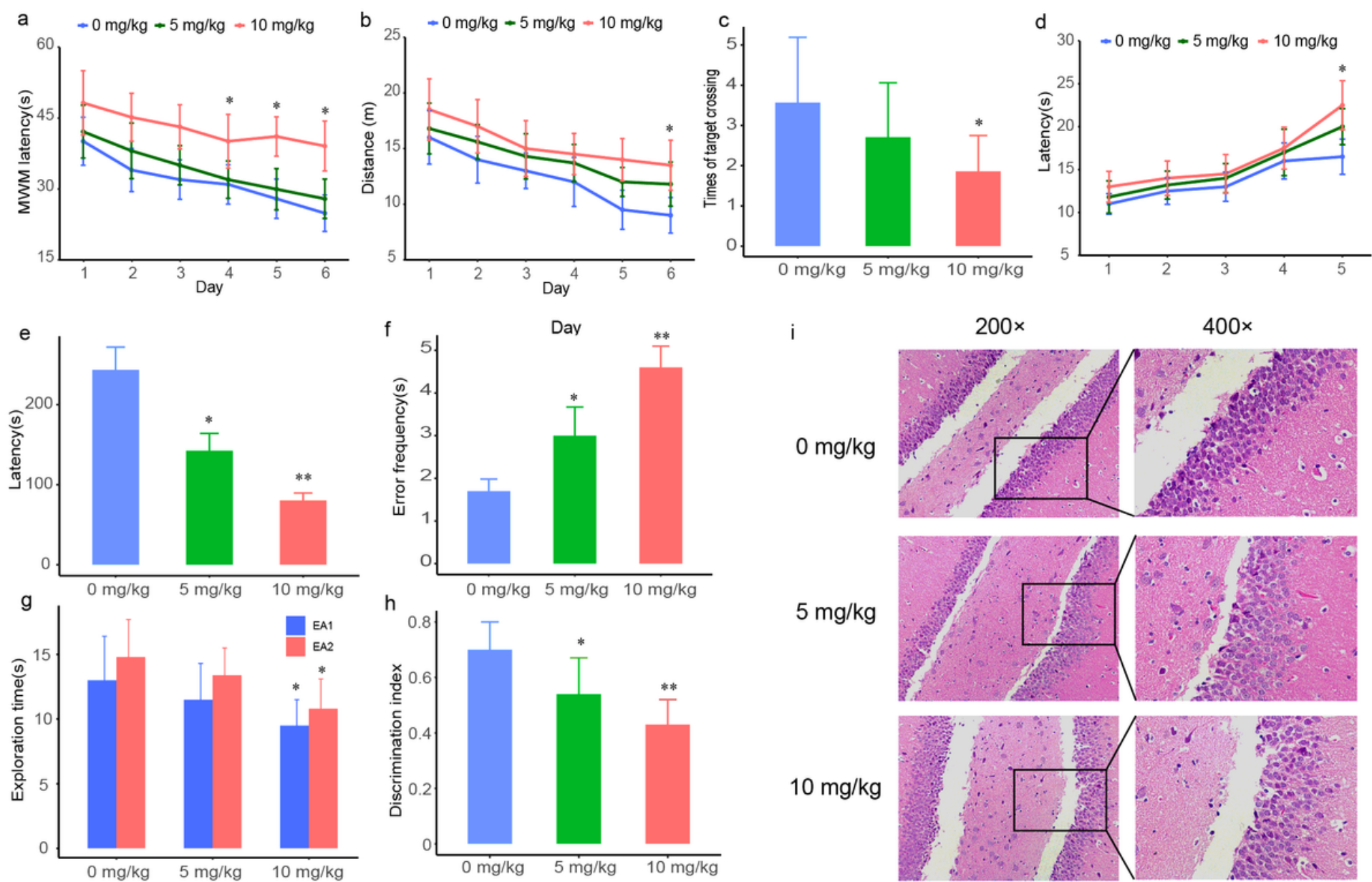

\section{Figure 2}

Behavioral tests in rats between the control group and acetochlor group. (a-c)The escape latency, the mean distance, and times of target crossing in the MWM test $(n=8)$. (d)The latency in the shuttle box test $(n=8)$. (e-f)The latency of step down and the error frequencies in the step-down test $(n=8)$. (g-h)The exploration time and discrimination index in the new object recognition test. (i)Effect of acetochlor exposure on the hippocampal histomorphology. Data were presented as mean $\pm S D\left({ }^{*} P<0.05,{ }^{\star \star} P<0.01\right)$. 

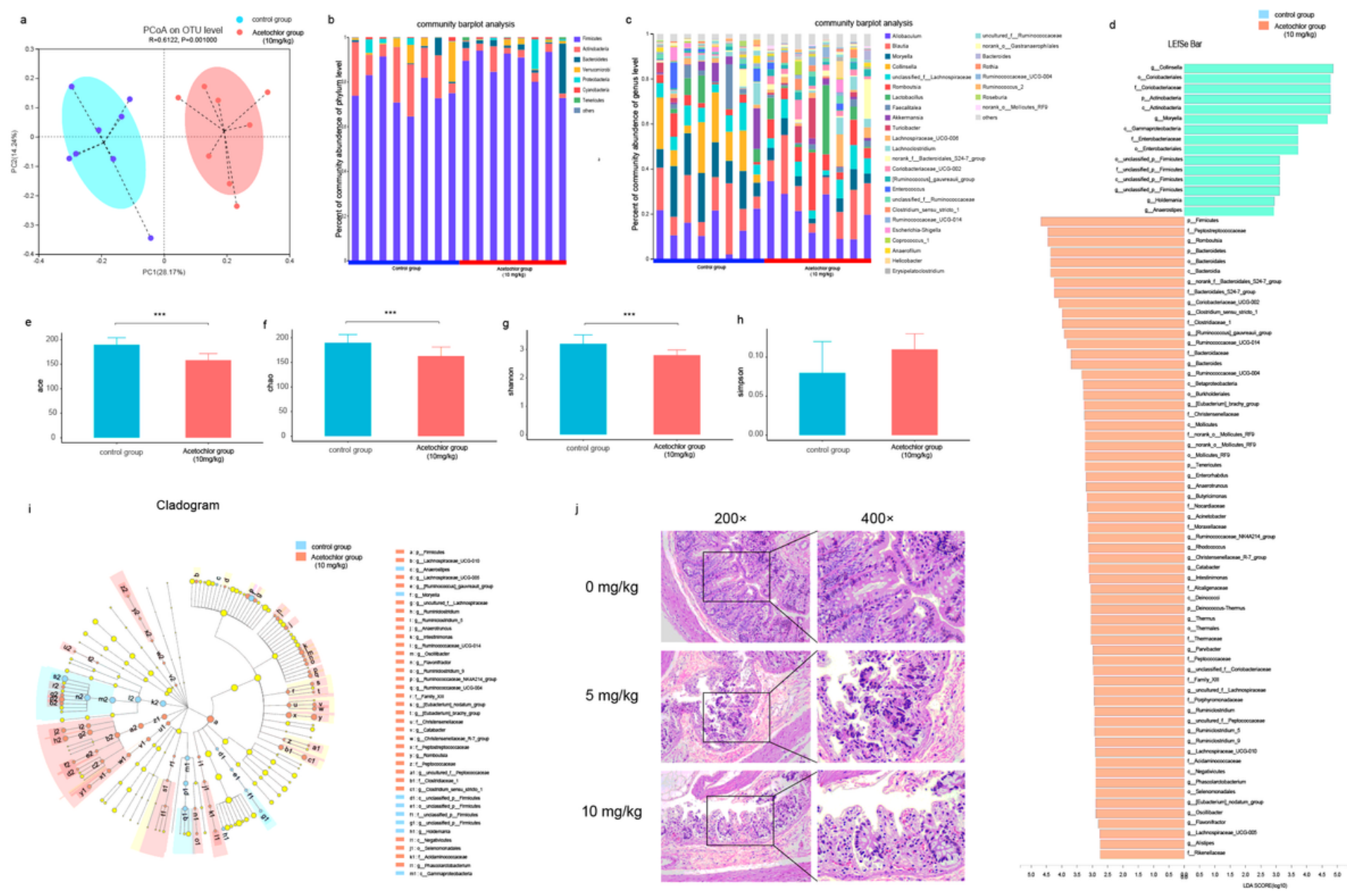

Figure 3

(a)PCoA plot based on weighted unifrac Distances. (b)Composition of the microbial community at the phylum level. (c)Composition of the microbial community at the genus level. (e-h)Alpha diversity of gut microbiota from both groups by Ace index, Shannon index, Chao1 index, and Simpson index.

$(\mathrm{d}, \mathrm{i})$ Differentially abundant taxa of gut microbiota between control and acetochlor-exposed rats. (j)Effect of acetochlor exposure on the colonic histomorphology. Data were presented as mean $\pm S D(\star \star \star P<0.001)$ $(n=6)$. 

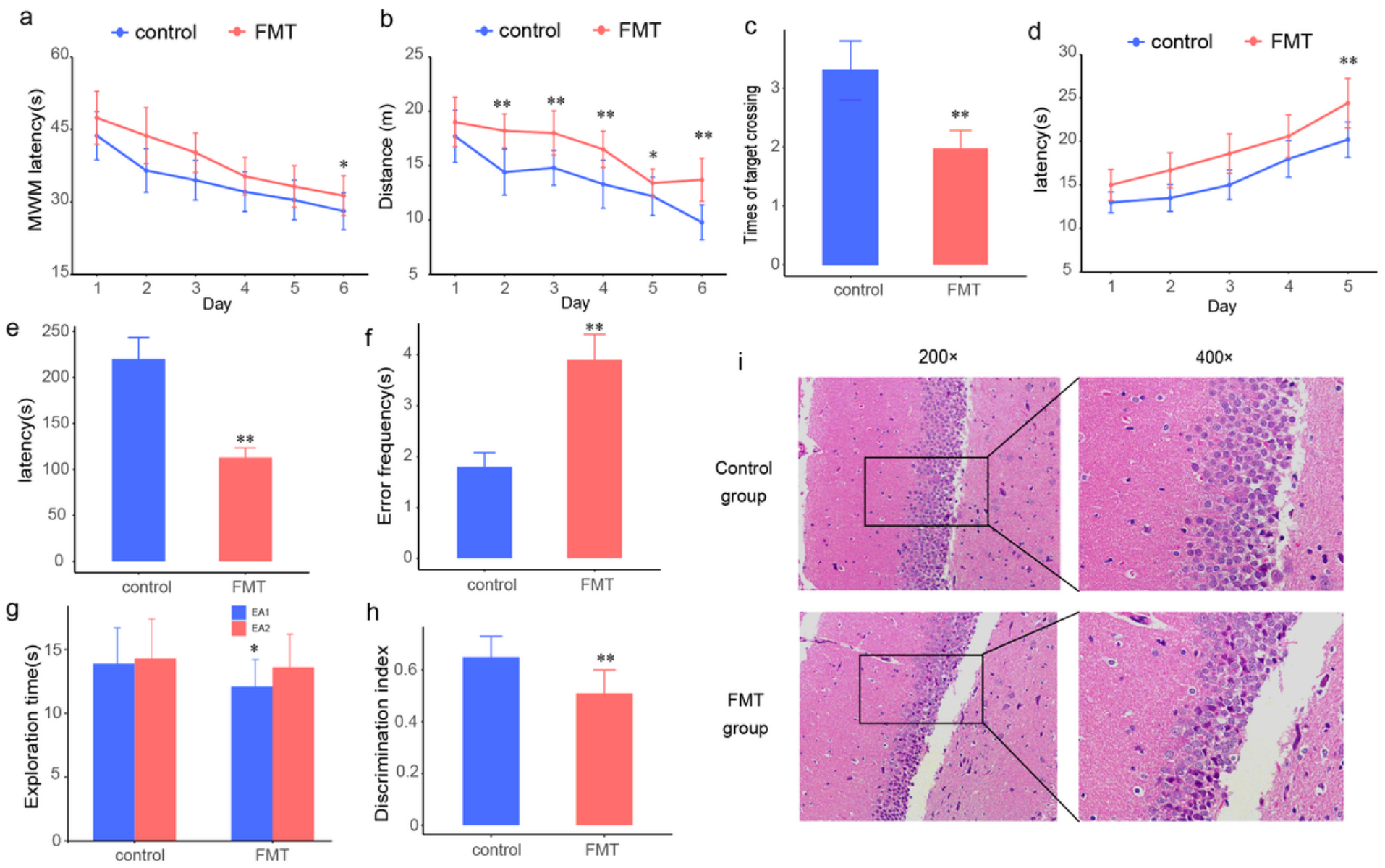

\section{Figure 4}

Behavioral tests in rats received fecal microbiota transplant or vehicle gavage. (a-c)The escape latency, the mean distance, and times of target crossing in the MWM test $(n=8)$. (d)The latency in the shuttle box test $(n=8)$. (e-f)The latency of step down and the error frequencies in the step-down test $(n=8)$. $(g-h)$ The exploration time and discrimination index in the new object recognition test. (i)Effect of FMT on the hippocampal histomorphology. Data were presented as mean $\pm S D\left({ }^{*} P<0.05,{ }^{*} P<0.01\right)$. 

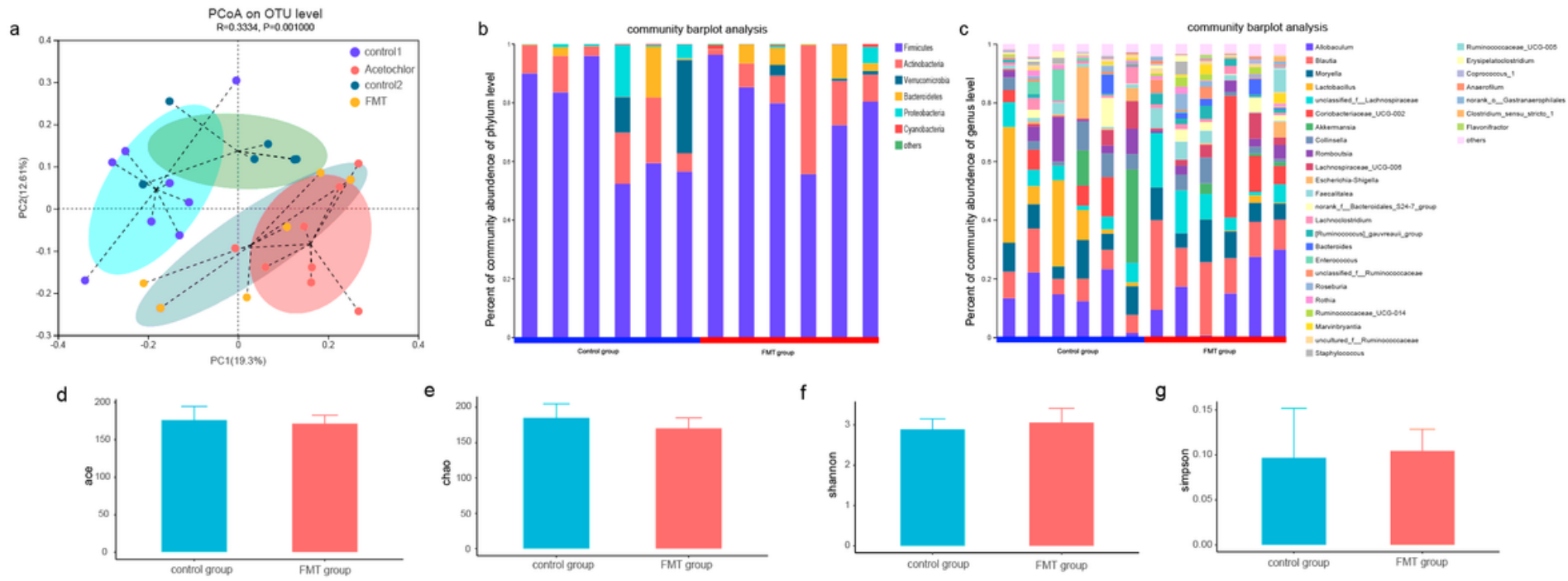

g
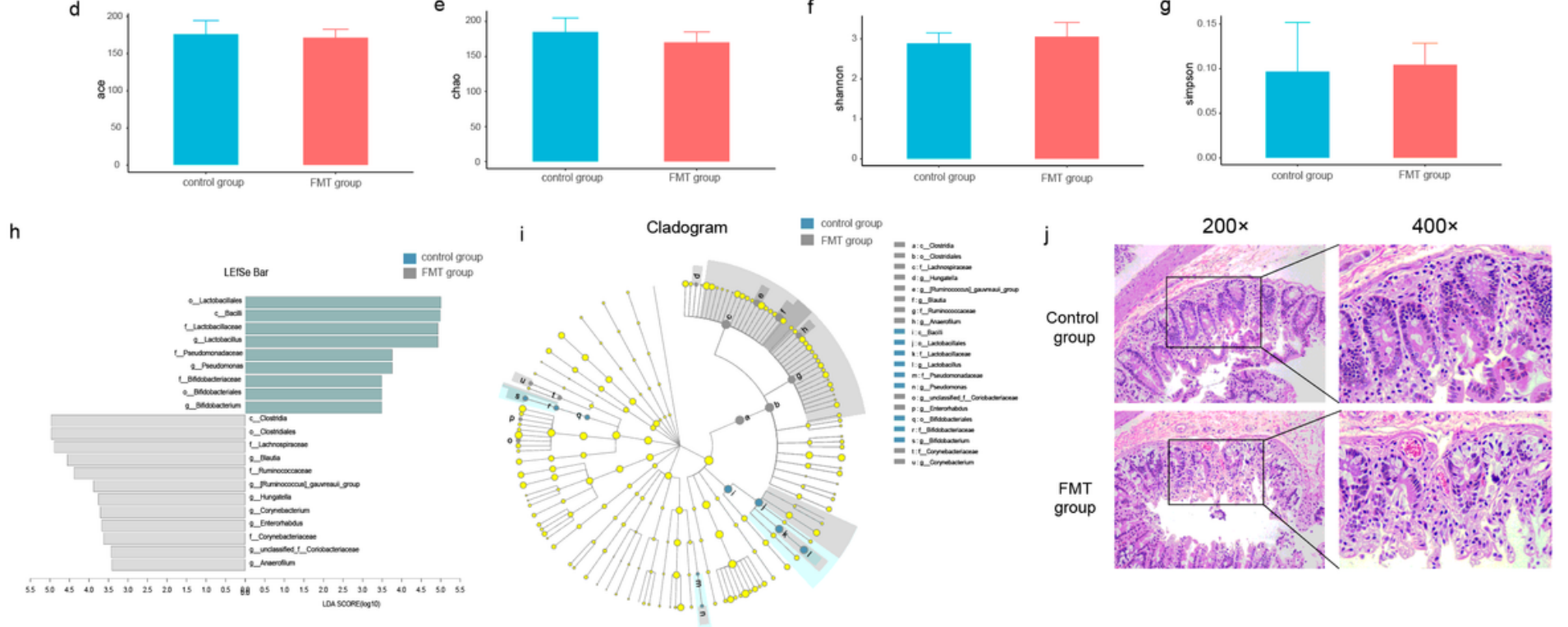

Figure 5

(a)PCoA plot based on weighted unifrac Distances. (b)Composition of the microbial community at the phylum level. (c)Composition of the microbial community at the genus level. (d-g)Alpha diversity of gut microbiota from both groups by Ace index, Shannon index, Chao1 index, and Simpson index.

$(h, i)$ Differentially abundant taxa of gut microbiota between control and FMT rats. (j)Effect of FMT on the colonic histomorphology. Data were presented as mean $\pm S D(n=6)$. 

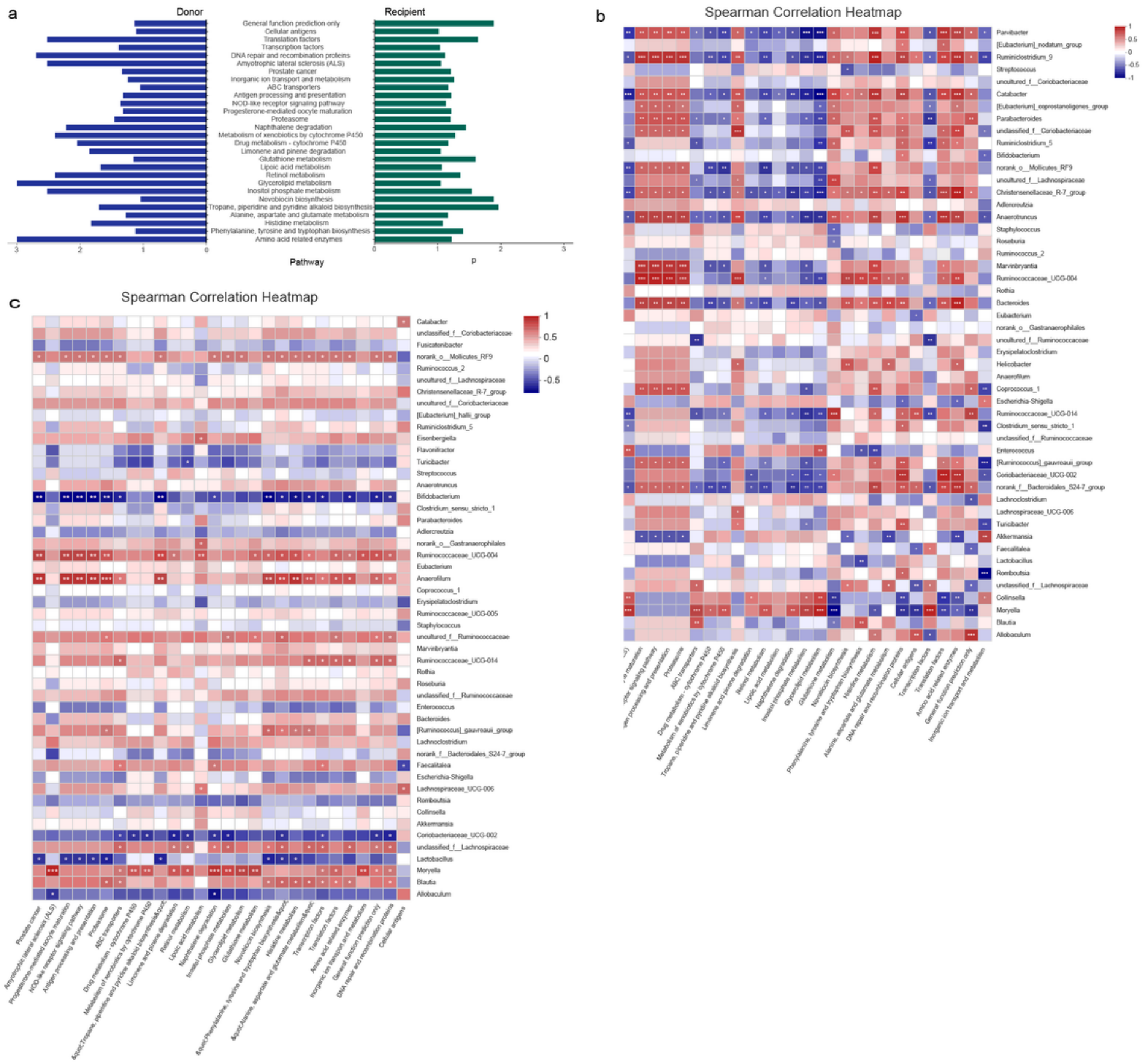

Figure 6

(a)Mutual differential pathways in both donor and recipient rats. $(b, c)$ The relationship between mutual differential pathways and top 50 species differential gut microbiome in donor and recipient rats. 
a

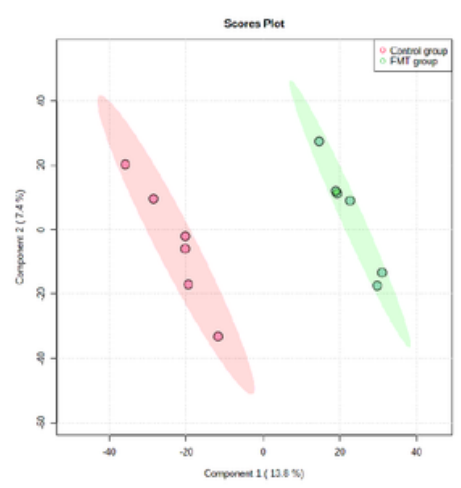

d

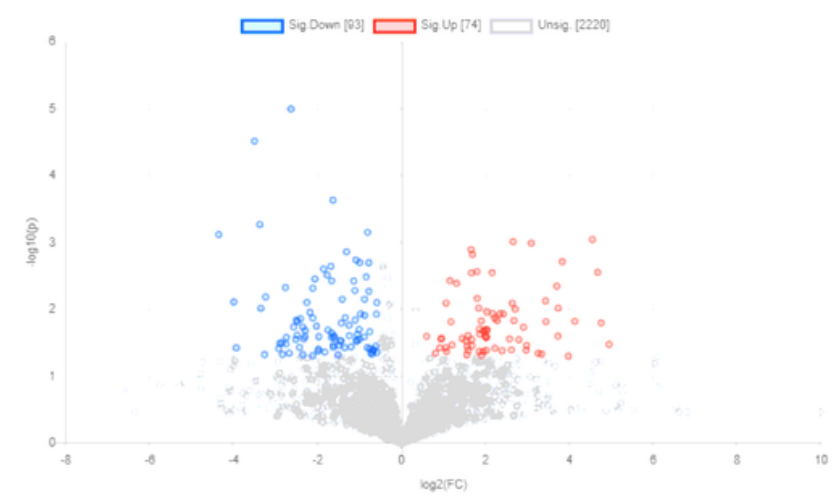

C

b

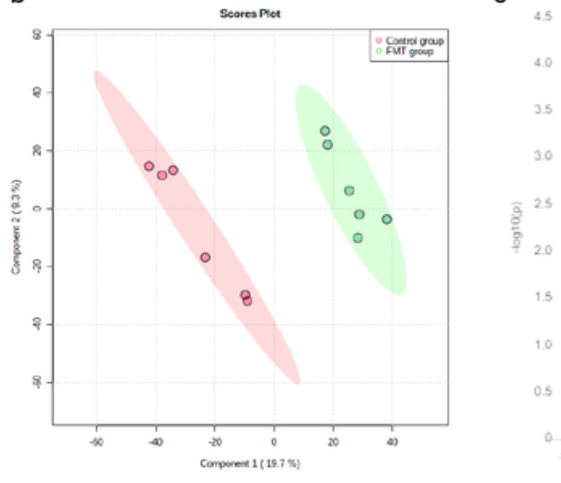

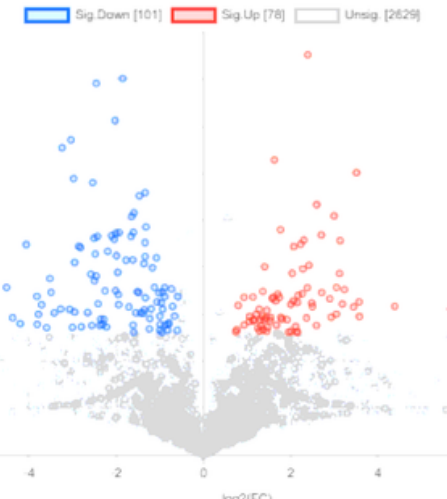

e

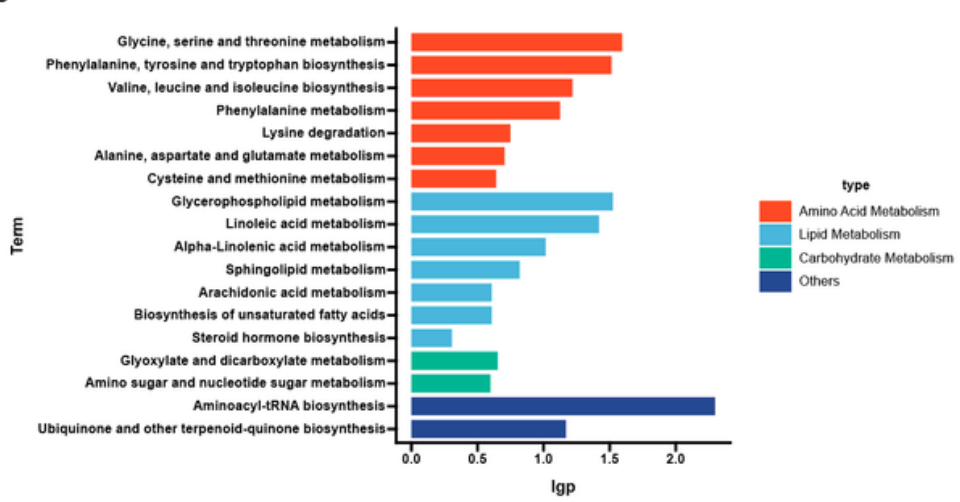

Figure 7

PLS-DA scores plot of hippocampus samples from control(Red) and FMT(green) groups in positive(a) and negative(b) ion mode. Volcano Plot showed differential metabolites in positive(c) and negative(d) ion mode. (e)Significantly altered pathways in FMT group $(n=6)$. 

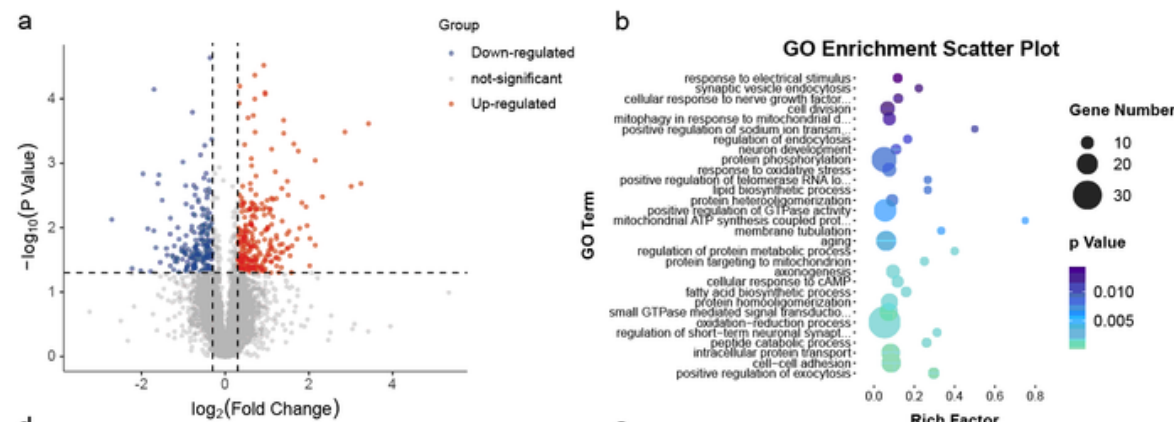

c
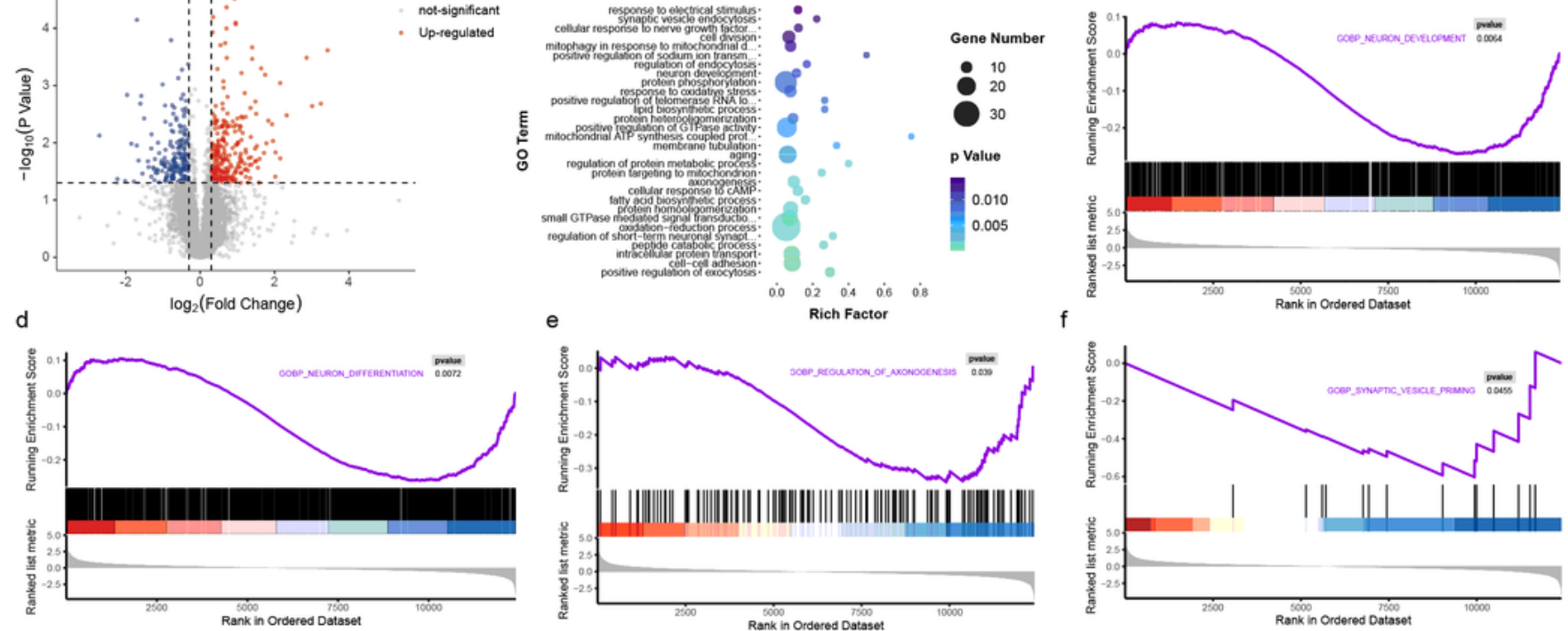

g KEGG Enrichment Bar Plot

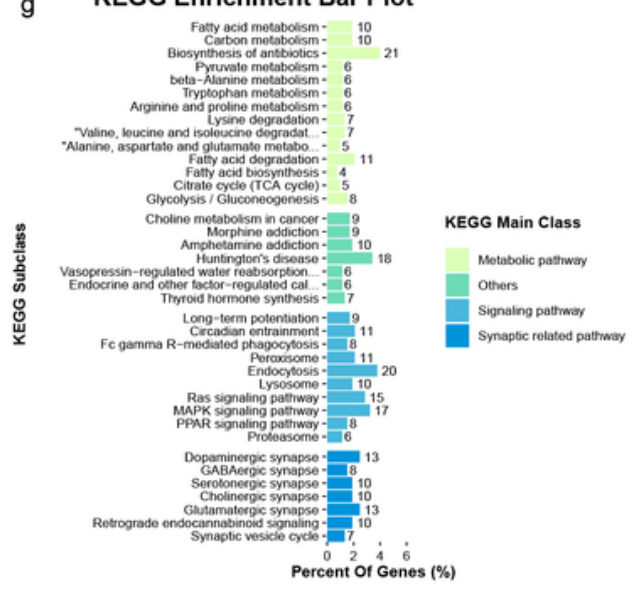

$\mathrm{h}$

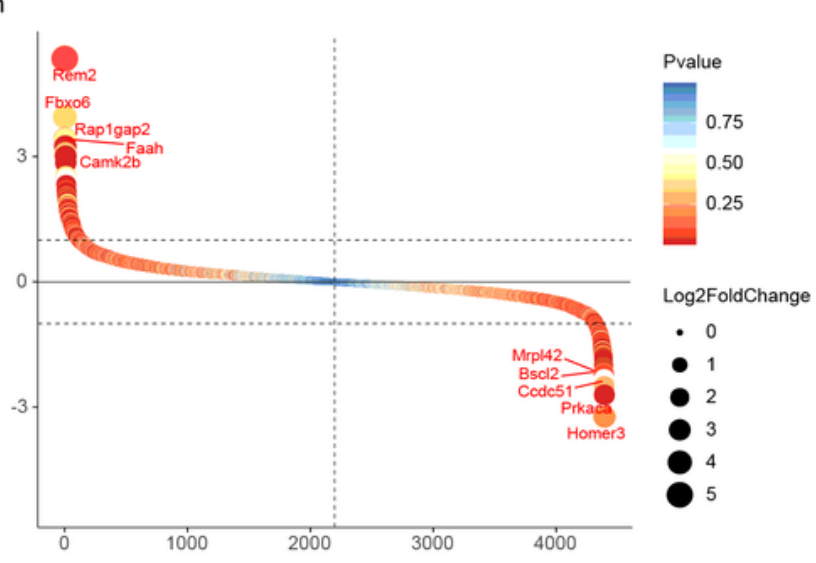

।

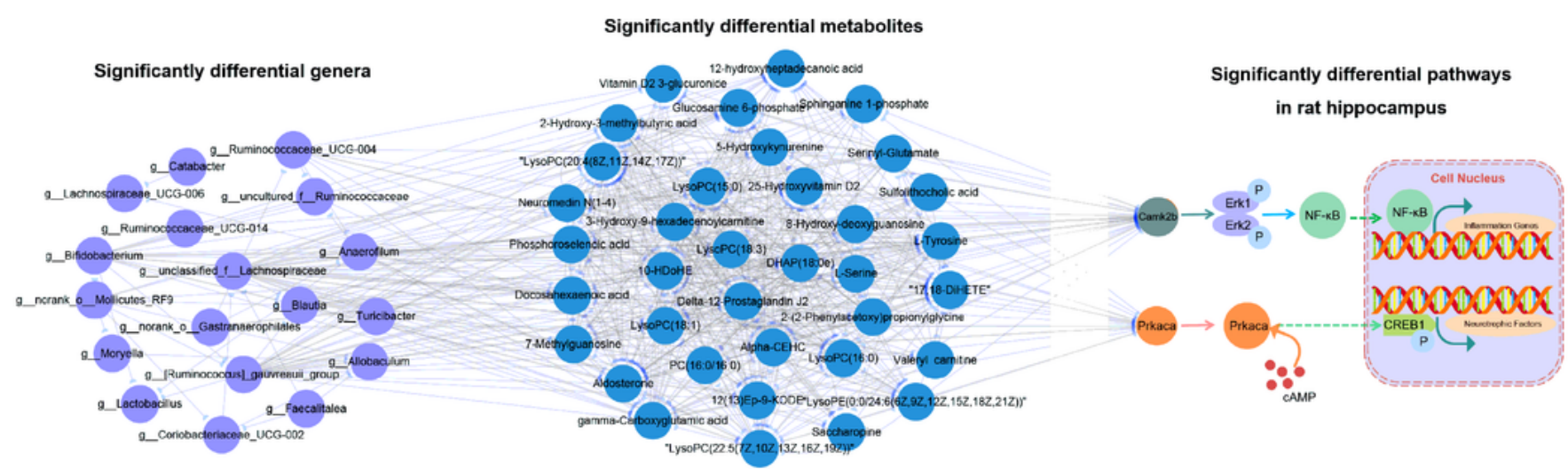

Figure 8

Differential expression proteins in control and FMT groups. (a)Volcano plot for differentially expressed proteins in rat hippocampus; (b)Biological processes for differentially expressed proteins; (c-f)Enrichment plots for nervous system related pathways obtained by the GSEA; (g)KEGG pathway for differentially expressed proteins; (h)S-shape figure showed the proteins with greater fold-change; (i)Network graph showed the relationship among differential genera, metabolites, proteins. 
Control group

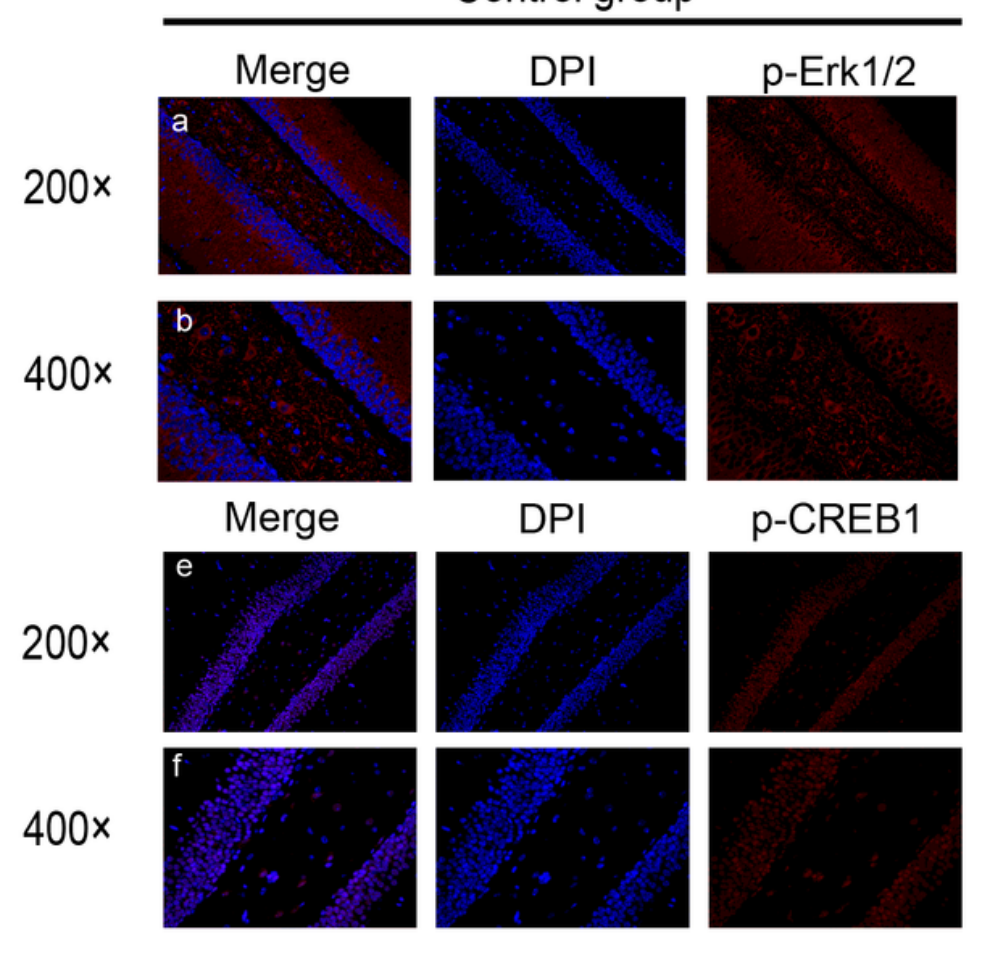

FMT group
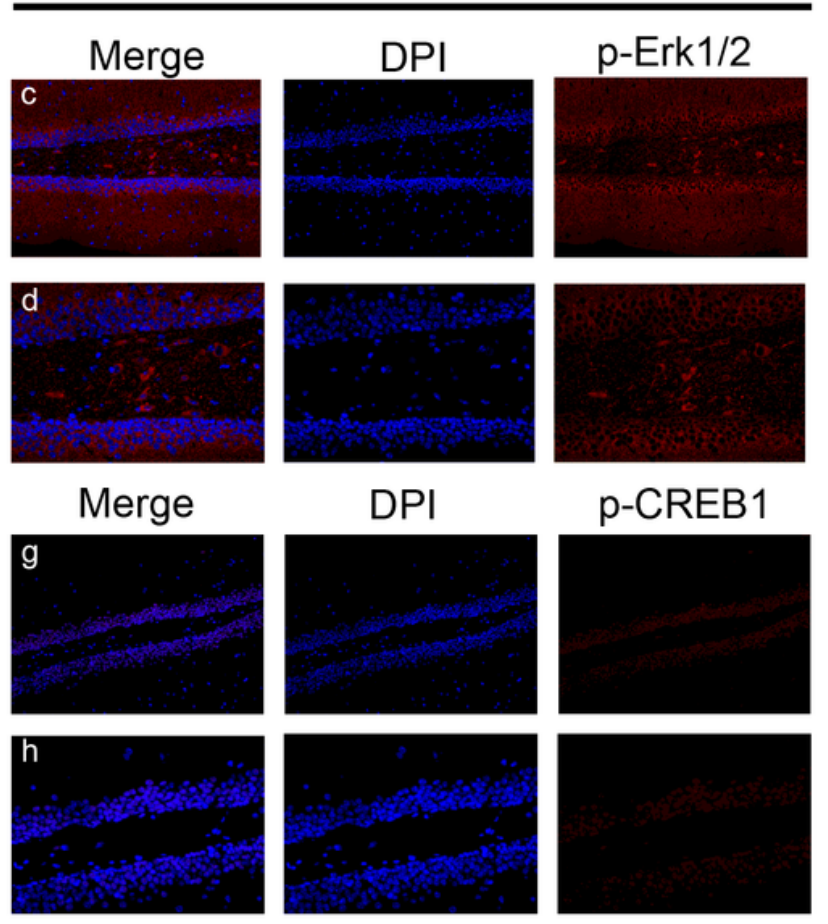

DPI
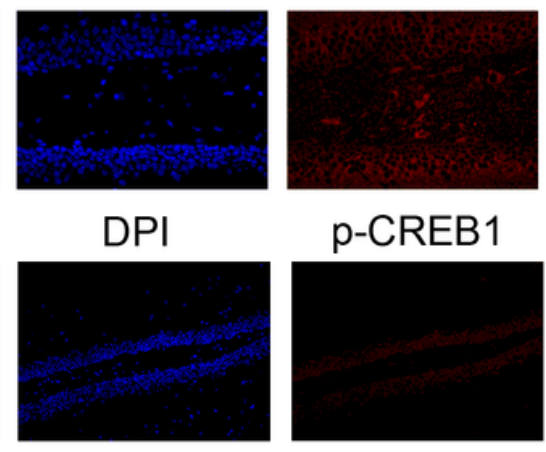

p-CREB1
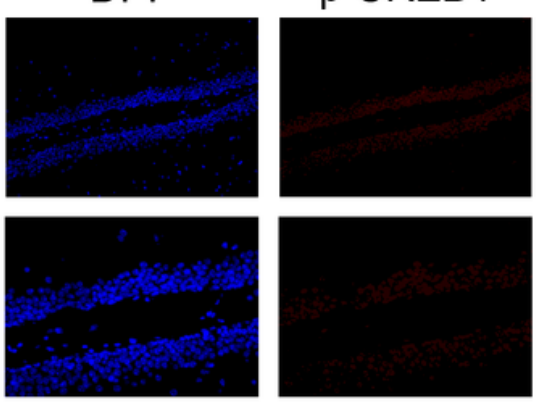
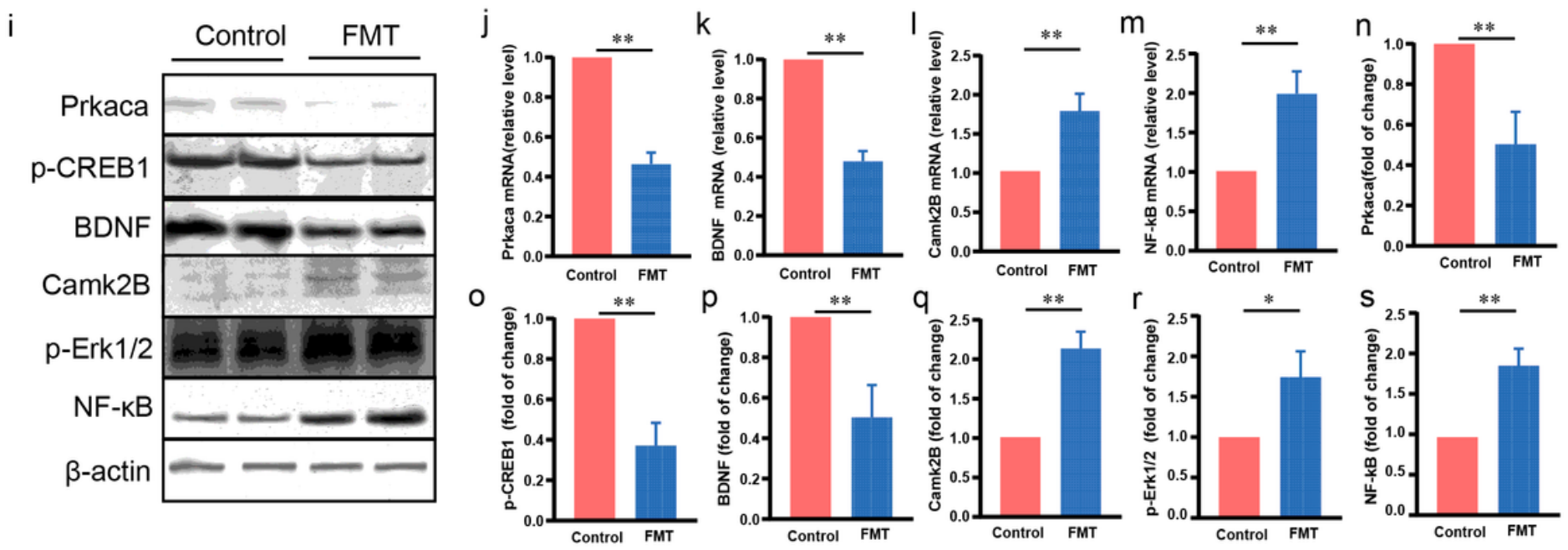

\section{Figure 9}

Representative images of p-Erk1/2(a-d) and p-CREB1(e-h) proteins in immunofluorescence analysis $(n=6)$; Effect of FMT on the mRNA levels of Prkaca(j), BDNF(k), Camk2B(I), NF-kB(m). Effect of FMT on the protein levels of Prkaca(n), p-CREB1(o), BDNF/2(p), Camk2B(q), p-Erk1/2(r), and NF-kB(s) $(n=6)$. Representative western blots of all proteins above are shown(i). Each column represents the mean $\pm S D(* P<0.05, * * P<0.01)$.

\section{Supplementary Files}

This is a list of supplementary files associated with this preprint. Click to download. 
- supplementarytable1.xlsx

- supplementarytable2.xlsx

- supplementarytable3.xlsx 